\title{
Infrared Coagulation for Treatment of Internal Hemorrhoids in Otherwise Healthy Patients and Specific Condition (A 5 Year Follow Up)
}

\section{Seyed Reza Fatemi ${ }^{*}$, Behzad Hatami ${ }^{1}$, Naghmeh Salarieh ${ }^{1}$, Azam Farahani $^{1}$, Seyed Ali Fatemi ${ }^{2}$, Sania Alizadeh ${ }^{2}$ and Mahdi Heydari Monfared $^{1}$}

${ }^{1}$ Gastroenterology and Liver Diseases Research Center, Research Institute for Gastroenterology and Liver Diseases, Shahid Beheshti University of Medical Sciences, Tehran, Iran

${ }^{2}$ Life Science, Biology, Student, University of Toronto, Canada

*Corresponding Author: Seyed Reza Fatemi, Gastroenterology and Liver Diseases

Research Center, Research Institute for Gastroenterology and Liver Diseases, Shahid Beheshti University of Medical Sciences, Tehran, Iran.
Received: June 16, 2021

Published: June 23, 2021

(C) All rights are reserved by Seyed Reza

Fatemi., et al.

\begin{abstract}
Background: Infrared coagulation (IRC) is one of nonsurgical office-based modalities for treatment of internal hemorrhoids. Although until recently, rubber band ligation (RBL) has mainly been considered as first-line therapy, it sounds that IRC can be used as first choice in treatment of grades I-III internal hemorrhoids, especially in certain conditions including pregnant and cirrhotic patients.

Methods: Our study was conducted prospectively and 220 patients including 190 otherwise healthy, 20 cirrhotic Child-Pugh class B or C and 10 pregnant patients, $46 \%$ female and 53\% male, with symptomatic internal hemorrhoids grades II or III underwent IRC in total 660 sittings over a 3- month period of treatment. Early and late complications and outcome of patients were monitored during a period of 5-year follow-up.

Results: Out of 220 patients just $85.8 \%$ experienced some short time post-procedure symptoms. The most common complaint was feeling of mild pressure or discomfort in anal canal (42.7\%) occurred within 24 hours to 10 days after procedure and completely resolved thereafter. The other complaint was trivial bleeding during defecation (23.2\%) for few days after procedure. The post procedure complaints in pregnant and cirrhotic patients were not different from otherwise healthy patients. Among 220 patients treated by IRC, only 33 patients (15\%) experienced recurrence of symptoms predominantly as rectal bleeding during long-term follow-up. The majority of relapses occurred within one year after procedure (81.8\%). Unexpectedly, the relapse rate in pregnant and cirrhotic patients were similar to otherwise healthy patients. Overall success rate of this modality for treatment of early internal hemorrhoids in long-term follow-up was $85 \%$.

Conclusion: IRC is a simple, swift, convenient and repeatable procedure with good safety index and high success rate for treatment of grades I-III internal hemorrhoids that can be conducted in outpatient setting for otherwise healthy patients as well as pregnant and cirrhotic patients. It can even be regarded as first -line therapy.
\end{abstract}

Keywords: Internal Hemorrhoids; Infrared Coagulation; Rectal Bleeding

\section{Introduction}

Hemorrhoids are arteriovenous vascular plexuses that lie in the distal rectum and anal canal. They become symptomatic when en- larged, inflamed, thrombosed, or prolapsed. Hemorrhoid disease is one of the most common anorectal diseases which present with a variety of symptoms including a prolapsed mass, painless bleeding, 
discomfort, fecal soiling, pruritus ani, and pain due to thrombosis [1-12]. Although these symptoms may strongly suggest the diagnosis, it is necessary to perform flexible sigmoidoscopy or colonoscopy in patients who present with bleeding to refute potential neoplastic sources as a source of the patients symptoms [13-15].

Hemorrhoids are categorized according to their position in relation to the dentate line. Those which located proximal to the dentate line are internal hemorrhoids and ones which located distal to the dentate line are considered as external hemorrhoids [16,17]. Internal hemorrhoids are further graded based on protrusion and reducibility (Goligher's classification) into Grade I: hemorrhoids with bleeding but without prolapse; Grade II: hemorrhoids that prolapse upon defecation and spontaneously reduce; Grade III: hemorrhoids requiring manual reduction of the prolapsed tissue and Grade IV* hemorrhoids which are not reducible. External hemorrhoids are not graded $[1,2,7,8,11]$. There are three treatment strategies for hemorrhoids including conservative (medical) treatment, minimally invasive office-based procedures and surgical therapy [9-12].

Conservative measures are effective in most symptomatic patients. Dietary and lifestyle modifications constitute the most important part of conservative treatment. This usually consists of increasing dietary fiber (up to 20 to $30 \mathrm{~g} /$ day) and oral liquid intake, the avoidance of straining and warm sitz baths two to three times per day $[18,19]$. If conservative approach fails, most patients with symptomatic grade I, II, and III internal hemorrhoids may be managed with nonsurgical office-based procedures. These include rubber band ligation (RBL), Infrared coagulation (IRC), bipolar diathermy (Bicap), laser photocoagulation, sclerotherapy, and cryosurgery [20-24]. Eventually, surgery is indicated in small number of patients with failed medical and non-operative therapy, symptomatic third- degree, fourth-degree or mixed internal and external hemorrhoids, symptomatic hemorrhoids with concomitant rectal pathology requiring surgery, and patient preference [1,2,9-13,25].

IRC technique was first described by Neiger in 1979 [4]. IRC uses infrared light to cause thrombosis, scarring and subsequent shrinkage of the hemorrhoidal tissue. An infrared device (Figure 1) is made of a light generator and a probe that delivers infrared energy into the tissue [26].

After inserting the probe through an anoscopy, three to five time, 1- to 1.5- second pulses of the infrared light are applied directly onto the normal mucosa proximal to the hemorrhoidal tissue, not the hemorrhoid itself. This causes protein coagulation with 3-mm wide and 3-mm deep (Figure 2). Only one or two hemorrhoids should be treated per session. The procedure can be repeated every
2-4 weeks for total of two or three sessions if further treatments necessitate $[1,3,4,11]$. IRC is approved by the U.S food and drug Administration (FDA) for treatment of grade I, II, and III internal hemorrhoids [11,27]. RCTS (randomized controlled trials) showed that IRC had higher primary success rate than RBL $[31,32,34]$. Nikravsh M., et al. [33] found a significant lower adverse event rate (bleeding and pain) and recurrent rate for IRC in compared IRC and closed hemorrhoidectomy in treatment internal hemorrhoid.
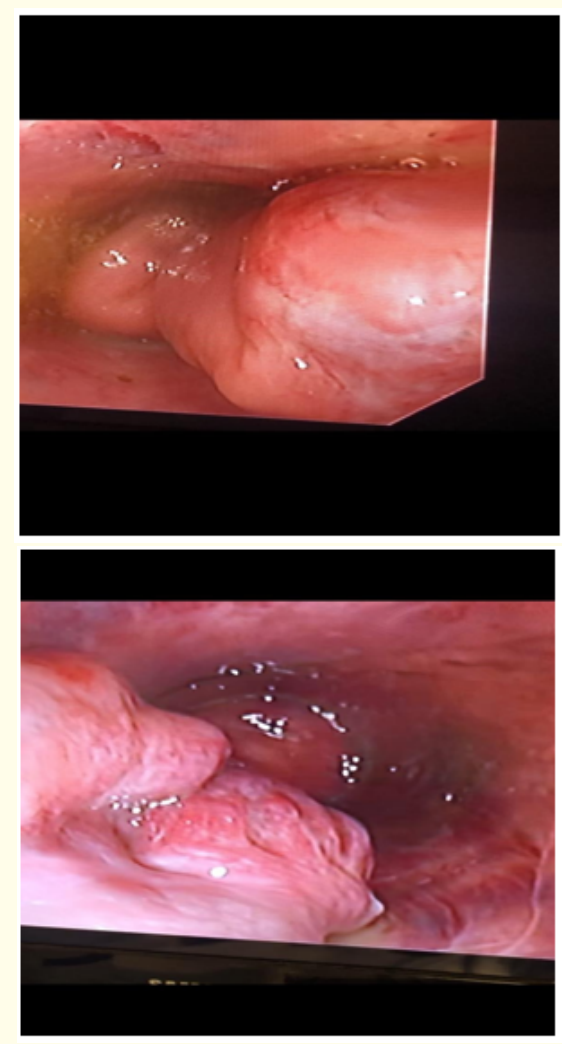

Figure 1: Internal hemorrhoids.

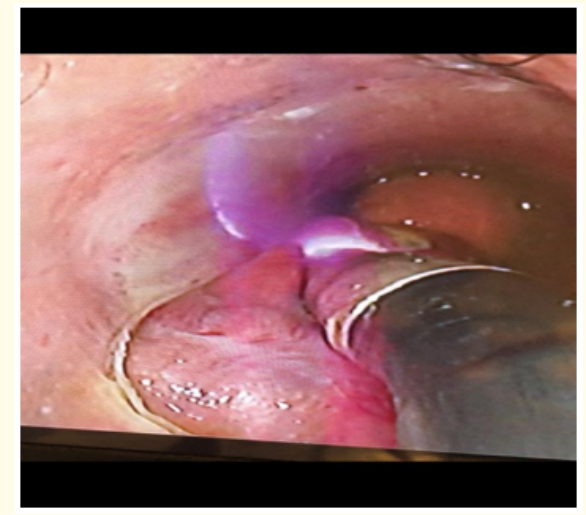



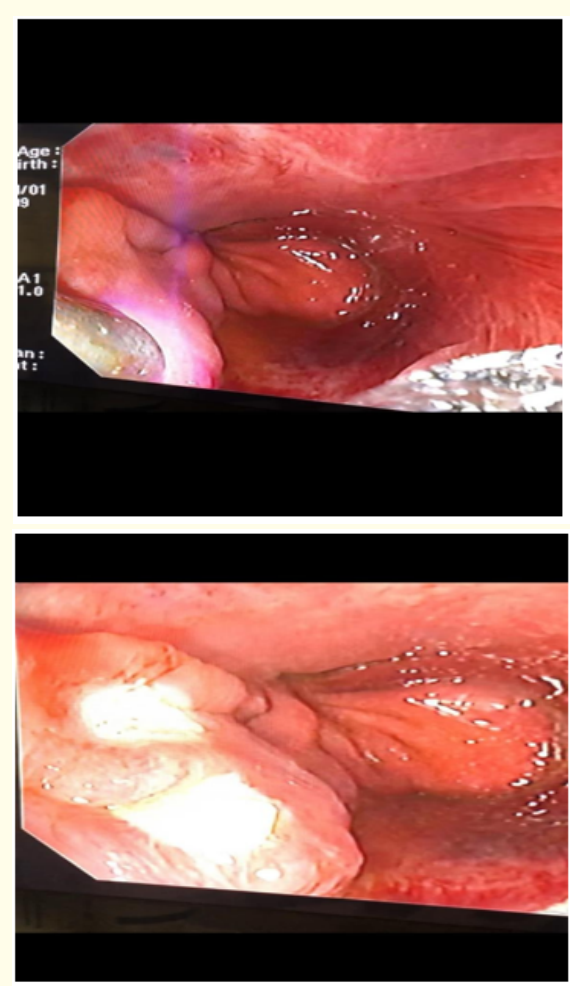

Figure 2: Infrared coagulation of internal hemorrhoid.

\section{Aims and Objectives}

The aim of current study was to evaluate efficacy and outcome of IRC in treatment of internal hemorrhoids in otherwise healthy patients and in certain conditions including pregnant and cirrhotic patients.

\section{Patients and Methods}

The present study was conducted prospectively. The total of 220 patients with symptomatic internal hemorrhoids were scheduled for treatment with IRC. Inclusion criteria were patients aged 18 81 years with internals hemorrhoids grades I to III presented with symptoms that can be attributed to hemorrhoidal disease including rectal bleeding, tissue prolapse, anal discomfort or itching and fecal soilage.

Patients with grade IV internal hemorrhoids, mixed internal and external hemorrhoids, infective anorectal pathologies such as proctitis and cryptitis, fissures and anal ulcers, and coagulation disorders were considered as exclusion criteria. Moreover, pregnant women less than 12 weeks and patients refused to sign the informed consent were omitted from the study.
Each patient underwent a comprehensive history and physical examination. Patients underwent a total of 660 IRC treatments in three sessions over a 3-month period. Flexible sigmoidoscopy or colonoscopy was also performed to exclude other sources of bleeding. Patients' data including age, sex, hemorrhoidal grade and symptoms were collected.

Informed consent was obtained from all participants prior to performing the procedure. Treatment was done by (Lomotec, Germany) IRC device with anesthesia. Then, all the patients were observed for post-procedure complications including pain, bleeding, discharge, and infection in very early period within 24 hours to 10 days after that and in long-term over a 60 -month period. Finally, the results were analyzed statistically by SPSS statistics 21 software. Descriptive statistics was used for analyzing of mean age in total studied population and frequency of different factors. Mean age in different groups and significant statistical relation evaluated by T-test and Chi-square, respectively. P-value lower than 0.05 was assumed as significant.

\section{Results}

Out of a total of 310 patients with mild complaints of internal hemorrhoids, 220 patients were qualified for the study and assigned prospectively to treatment with infrared coagulation. Among these patients, 190 were otherwise healthy without any significant disorder, 20 patients had Child-Pugh class B or C cirrhosis and 10 patients were pregnant in their second or third trimesters. The post procedure follow-ups were carried out at 1 week, 4 weeks, 12 months and then annually for a total of 5 years.

Out of 220 patients $/ 102(46.4 \%)$ patients were male with mean age of (35.82 \pm 10.28$)$ years and $118(53.6 \%)$ were female with mean age of (32.23 \pm 10.39$)$ years. Ten $(4.5 \%)$ patients were pregnant and 20 (9.09\%) patients were cirrhotic. Ninety-nine (45\%) patients had family history of hemorrhoidal disease and 165 (75\%) patients carried history of longstanding constipation before treatment. All the patients had grade II or grade III symptomatic internal hemorrhoids. Among the presenting symptoms prior to treatment, which made patients seek medical attention, rectal bleeding was the most common (145 patients, 65.9\%). Other symptoms in order of frequency were tissue prolapse $(53.2 \%)$, anal discomfort (49.1\%), itching (26.4\%) and fecal seepage (11.4\%).

The post -procedure complaints over a 24-hour to 10-day period are shown in table 1 . Only $65.9 \%$ of patients experienced complications including anal discomfort and bleeding. They never experienced severe pain unresponsive to simple analgesics or 
heavy rectal bleeding. There were not more complications such as bleeding and pain in cirrhotic and pregnant patients than otherwise healthy ones.

\begin{tabular}{|l|c|c|}
\hline Post-IRC complications & Frequency (\%) & P-value \\
\hline Bleeding (within 10 days) & $51(23.2)$ & 0.17 \\
\hline $\begin{array}{l}\text { Pain or discomfort } \\
\text { (within 10 days) }\end{array}$ & $94(42.7)$ & 0.97 \\
\hline Infection & $0(0 \%)$ & - \\
\hline Perforation & $0(0 \%)$ & - \\
\hline
\end{tabular}

Table 1: Post-IRC complications.

Recurrence in our study was merely defined based on returning back of symptoms. Over a follow up period ranging from 12 to 60 months, out of 220 patients, 33 (15\%) patients developed recurrence. Out of 33 patients, 27 (81.8\%) patients within one year after IRC and $6(18.2 \%)$ patients beyond the first-year experienced recurrence of symptoms in the form of predominately bleeding. These patients successfully underwent IRC again. There was no significant correlation between recurrence and presenting symptoms such as pain or discomfort ( $p=0.70)$, itching ( $p=0.83$ ), bleeding $(p=1.00)$, discharge $(p=0.55)$ or prolapse $(p=0.35)$. Also, there was no meaningful correlation between recurrence and post procedure pain or bleeding $(\mathrm{p}=0.54)$. Moreover, recurrence was not correlated with hemorrhoidal grade ( $p$-value $<0.001$ ), although patients with grade III internal hemorrhoids had more relapse rate than patients with grade II; Further details are shown in table 2. After IRC, there was a significant relationship between recurrence and constipation (Table 2), but not between recurrence and patient gender, although recurrence was more frequent among females.

\begin{tabular}{|c|c|c|c|}
\hline & $\begin{array}{l}\text { Patients with } \\
\text { recurrence }\end{array}$ & $\begin{array}{l}\text { Patients without } \\
\text { recurrence }\end{array}$ & p-value \\
\hline \multicolumn{4}{|l|}{ Grade } \\
\hline Grade II & 17 (8.9\%) & $173(91.1 \%)$ & \multirow[t]{2}{*}{$<0.001$} \\
\hline Grade III & $16(53.3 \%)$ & $14(46.7 \%)$ & \\
\hline \multicolumn{4}{|l|}{ Constipation } \\
\hline $\begin{array}{l}\text { Patients with } \\
\text { constipation }\end{array}$ & $20(32.3 \%)$ & $42(67.7 \%)$ & \multirow[t]{2}{*}{$<0.001$} \\
\hline $\begin{array}{l}\text { Patients without } \\
\text { constipation }\end{array}$ & $13(8.2 \%)$ & $145(91.8 \%)$ & \\
\hline
\end{tabular}

Table 2: Factors may influence recurrence.

\section{Discussion}

IRC is one of the non-operative modalities for treatment of internal hemorrhoids. According to most comparative studies, RBL is the first-line therapy for early hemorrhoids. IRC is generally regarded as an alternative to RBL [7,8,28-30].

In this study, we aimed to evaluate the complications, outcome and success rate of IRC in treating internal hemorrhoids in otherwise healthy patients, as well as pregnant and cirrhotic patients.

In our study, the most common post procedure complications were localized pain (42.7\%) and mild bleeding (23.2\%). Pain is described by patients as some feeling of pressure, but not severe pain, in anal canal that persisted for a few days after IRC and relieved spontaneously or by limited use of topical NSAIDs for 24 72 hours. The bleeding was associated with defecation. There was no heavy bleeding to need surgical intervention, even in cirrhotic patients.

In study of Gupta PJ., et al. rectal bleeding after IRC was reported in $14 \%$ of patients. No severe pain were described following IRC [29].

In present study, recurrence rate was $15 \%$ and a vast majority of relapses were in the form of bleeding which occurred within one year after IRC.

According to two randomized controlled trials, Success rate of IRC for treatment of grade I and II bleeding hemorrhoids was $67 \%$ - $96 \%$ [4].

Based on a study by Gupta PJ., et al. in 2004, recurrence of bleeding after hemorrhoidal treatment with IRC was $12 \%$. Recurrence of prolapse was reported as $2 \%$ [29].

In a prospective clinical trial by John L Templeton., et al. IRC accompanied by a satisfactory outcome in $85 \%$ of patients. In a prospective randomized comparison of IRC and RBL in treatment of hemorrhoids, regardless of their grade, at one year follow-up, there was no significant difference in the symptomatic outcome of treatment between the two groups [30]. In a randomized controlled trial by Poen AC., et al. which compared rubber bad ligation versus IRC in the treatment of internal hemorrhoids, recurrent rate in RBL group was $18 \%$ and in IRC group was $20 \%$, however pain was more severe and more common after RBL [31]. In randomized clinical trial by Mohammad Afzal., et al. patients were followed for 12 months after hemorrhoidectomy with RBL and IRC, success rate was $88 \%$ in RBL and 94\% in RBL. intensity and duration of postoperative pain in the first week were greater in RBL than in IRC group [32]. In a study by Mohammadreza Nikshoar., et al. bleeding was $5 \%$ in IRC and 30\% in closed hemorrhoidectomy. Recurrent rate in IRC was $5 \%$ in IRC versus $20 \%$ in surgical group [33]. In clinical 
trial that was done by Mauricio Pichle R., et al. bleeding was found $34 \%$ in RBL and $16 \%$ after IRC, pain sensation was $52 \%$ during $52 \%$ and $39 \%$ in RBL. One month after treatment success rate was $73 \%$ in RBL and $72 \%$ in IRC [34].

To the best of our knowledge, there is no report of using IRC for treatment of internal hemorrhoids in cirrhotic and pregnant patients. However, our study showed that IRC was an effective and safe treatment option in these patients with the same recurrence rate compared to otherwise healthy patients.

\section{Conclusion}

IRC is a simple and convenient option of treatment of early internal hemorrhoids that can be conducted in office. It carries an acceptable safety index and a good success rate for treatment of grade I-III internal hemorrhoids and satisfies with patients so that IRC can be regarded as first-line therapy along with RBL. In addition, IRC is a safe and efficient option in pregnant and cirrhotic patients.

\section{Bibliography}

1. Halverson A. "Hemorrhoids". Clinics in Colon and Rectal Surgery 20.02 (2007): 077-085.

2. Sanchez C and Chinn BT. "Hemorrhoids". Clinics in Colon and Rectal Surgery 24.01 (2011): 005-013.

3. Singal R., et al. "An optimal painless treatment for early hemorrhoids; our experience in Government Medical College and Hospital". Journal of Medicine and Life 6.3 (2013): 302.

4. Čuk V., et al. "Where are we now in the treatment of hemorrhoids". Acta Medica Medianae 54.1 (2015): 97-106.

5. Gupta PJ. "Infrared Coagulation versus Rubber Band Ligation in Early Hemorrhoids; PAIN VS GAIN". Shiraz E-Medical Journal $5.2(2004)$.

6. Song S-G and Kim S-H. "Optimal treatment of symptomatic hemorrhoids". Journal of the Korean Society of Coloproctology 27.6 (2011): 277-281.

7. Sakr M and Saed K. "Recent advances in the management of hemorrhoids". World Journal of Surgical Procedures 4.3 (2014): 55-65.

8. McArthur BS and Karandikar S. "Current trends in the management of haemorrhoids". Bombay Hospital Journal 50 (2008): 343-348.

9. Ganz RA. "The evaluation and treatment of hemorrhoids: a guide for the gastroenterologist". Clinical Gastroenterology and Hepatology 11.6 (2013): 593-603.
10. McLemore EC., et al. "Novel endoscopic delivery modality of infrared coagulation therapy for internal hemorrhoids". Surgical Endoscopy 26.11 (2012): 3082-3087.

11. Siddiqui UD., et al. "Devices for the endoscopic treatment of hemorrhoids". Gastrointestinal Endoscopy 79.1 (2014): 8.

12. Zinberg SS., et al. "A personal experience in comparing three nonoperative techniques for treating internal hemorrhoids". The American Journal of Gastroenterology 84.5 (1989): 488492.

13. Moesgaard F., et al. "High-fiber diet reduces bleeding and pain in patients with hemorrhoids". Diseases of the Colon and Rectum 25.5 (1982): 454-456.

14. Csiky L. "Circular stapler for hemorrhoid operations". Google Patents (2010).

15. Piskun G. "Hemorrhoids treatment method and associated instrument assembly including anoscope and cofunctioning tissue occlusion device". Google Patents (2006).

16. Aigner F., et al. "The vascular nature of hemorrhoids". Journal of Gastrointestinal Surgery 10.7 (2006): 1044-1050.

17. Lohsiriwat V. "Hemorrhoids: from basic pathophysiology to clinical management". World Journal of Gastroenterology 18.17 (2012): 2009-20017.

18. Alonso-Coello P., et al. "Fiber for the treatment of hemorrhoids complications: a systematic review and meta-analysis". The American Journal of Gastroenterology 101.1 (2006): 181-188.

19. Perez-Miranda M., et al. "Effect of fiber supplements on internal bleeding hemorrhoids". Hepato-Gastroenterology 43.12 (1995): 1504-1507.

20. Bayer I., et al. "Rubber band ligation of hemorrhoids: convenient and economic treatment". Journal of Clinical Gastroenterology 23.1 (1996): 50-52.

21. Aghaei M., et al. "Comparative Study of Staging Rubber Band Ligation (RBL) and Hemorrhoidectomy in Treatment of Forth Degree Hemorrhoids". Annals of Colorectal Research 1.2 (2013): 62-66.

22. Aram FO. "Rubber Band Ligation for Hemorrhoids: an Office Experience”. Indian Journal of Surgery (2015): 1-4.

23. Lohsiriwat V. "Treatment of hemorrhoids: A coloproctologist's view". World Journal of Gastroenterology: WJG 21.31 (2015): 9245.

24. Johanson JF and Rimm A. "Optimal nonsurgical treatment of hemorrhoids: a comparative analysis of infrared coagulation, rubber band ligation and injection sclerotherapy". American Journal of Gastroenterology 87 (1992): 1601. 
25. Committee AT., et al. "The role of endoscopy in patients with anorectal disorders". Gastrointestinal Endoscopy 72.6 (2010): 1117-1123.

26. Leicester RJ., et al. "Infrared coagulation: a new treatment for hemorrhoids". Diseases of the Colon and Rectum 24.8 (1980): 602-605.

27. Salvati EP. "Nonoperative management of hemorrhoids". Diseases of the Colon and Rectum 42.8 (1999): 989-993.

28. Marques C., et al. "Early results of the treatment of internal hemorrhoid disease by infrared coagulation and elastic banding: a prospective randomized cross-over trial". Techniques in Coloproctology 10.4 (2006): 312-317.

29. Gupta PJ. "Infrared coagulation: a preferred option in treating early hemorrhoids". Acta Cirurgica Brasileira 19.1 (2004): 7478.

30. Templeton JL., et al. "Comparison of infrared coagulation and rubber band ligation for first and second degree haemorrhoids: a randomised prospective clinical trial". British Medical Journal 286.6375 (1983): 1387-1389.

31. Poen AC., et al. "Rubber bandligation versus infera red coagulation in the treatment of internal hemorrhoid: a randomized controlled trial". European Journal of Gastroenterology and Hepatology 12.5 (2000): 535-53.9.

32. Afzal M., et al. "Infrared coagulation versus rubber band ligation in treatment internal hemorrhoid: a randomised prospective study". Pakistan Armed Forces Medical Journal 59.2 (2009): 165-169.

33. Nikshoar MR., et al. "The Clinical Efficacy of Infrared Photocoagulation versus Closed Hemorrhoidectomy in Treatment of hemorrhoid". Journal of Lasers in Medical Sciences 9 (2018): 23-22.

34. Pichler Ricci M., et al. "Rubber band ligation and infrared photocoagulation for the outpatient treatment of hemorrhoid disease: a randomized controlled trial". Acta Cirúrgica Brasileira 23.1 (2008):102-106.

Volume 4 Issue 7 July 2021

(C) All rights are reserved by Seyed Reza Fatemi., et al. 